\title{
Is it time to modify the reverse cholesterol transport model?
}

\section{Commentary}

See related article, pages $1315-1320$

\author{
Alan R. Tall, Nan Wang, and Phillip Mucksavage \\ Columbia University, Department of Medicine, Division of Molecular Medicine, New York, New York, USA \\ Address correspondence to: Alan R. Tall, Columbia University, Department of Medicine, Division of Molecular Medicine, \\ New York, New York 10032, USA. Phone: (212) 305-4899; Fax: (212) 305-5052; E-mail: art1@columbia.edu.
}

J. Clin. Invest. 108:1273-1275 (2001). DOI:10.1172/JCI200114342.

ABCA1 was recently identified as the mutant molecule in Tangier disease, a condition associated with very low HDL, cholesteryl ester accumulation in tissue macrophages, and an apparent increased risk of atherosclerotic cardiovascular disease. ABCA1 is an ATP-binding cassette transporter that facilitates the addition of phospholipids and cholesterol to free apoA-I, initiating the formation of HDL (1). ABCA1 is upregulated in cholesterolloaded cells, as a result of activation of $\mathrm{LXR} / \mathrm{RXR}$ at the proximal promoter of the $A B C A 1$ gene $(2,3)$. It has been proposed that increased expression of ABCA1 in macrophages or other tissues could activate the excretion of cholesterol via HDL and retard the development of atherosclerosis $(3,4)$. While we await the results of atherosclerosis studies, two reports in the JCI on ABCA1 knockout (KO) mice are notable, since they address different aspects of this hypothesis and reach some unexpected conclusions that call into question the accepted view of reverse cholesterol transport.

\section{Macrophage ABCA1 contributes little to maintaining plasma HDL levels \\ ABCA1 KO mice have very low $\mathrm{HDL}$ levels and accumulate macrophage foam cells in the lung (5). In this issue of the JCI, Haghpassand et al. describe elegant studies in which they have esti- mated the contribution of macrophage ABCA1 to overall plasma HDL levels (6). These authors transplanted bone marrow from wild-type mice into ABCA1 KO mice and from ABCA1 KO mice into wild-type mice. In the former experiment, they found that apoA-I and HDL cholesterol were slightly but sig- nificantly increased, suggesting a minor contribution of macrophage ABCA 1 to plasma HDL levels. In the latter experi- ment, they could detect no contribu- tion at all; HDL levels were unchanged.}

These experiments underestimate the macrophage ABCA1 contribution to HDL, since only about $35 \%$ of liver macrophages were repopulated within the 14 weeks that the transplanted mice were studied. Nevertheless, it can be reasonably concluded that macrophage ABCA1 does not make a large contribution to HDL levels. This result probably reflects the widespread expression of ABCA1 in different cell types. In par- ticular, hepatocytes express ABCA1, and overexpression of ABCA1 in both liver and macrophages in ABCA1 transgenic mice appears to result in elevated HDL cholesterol and apoA-I levels (7). Presumably, the major contribution to HDL is from hepatic expression (Figure 1). Since ABCA1 binds and lipidates free apoA-I, but not bulk HDL (8), these results imply that hepatic $\mathrm{ABCA} 1$ gives rise to bulk HDL particles, independent

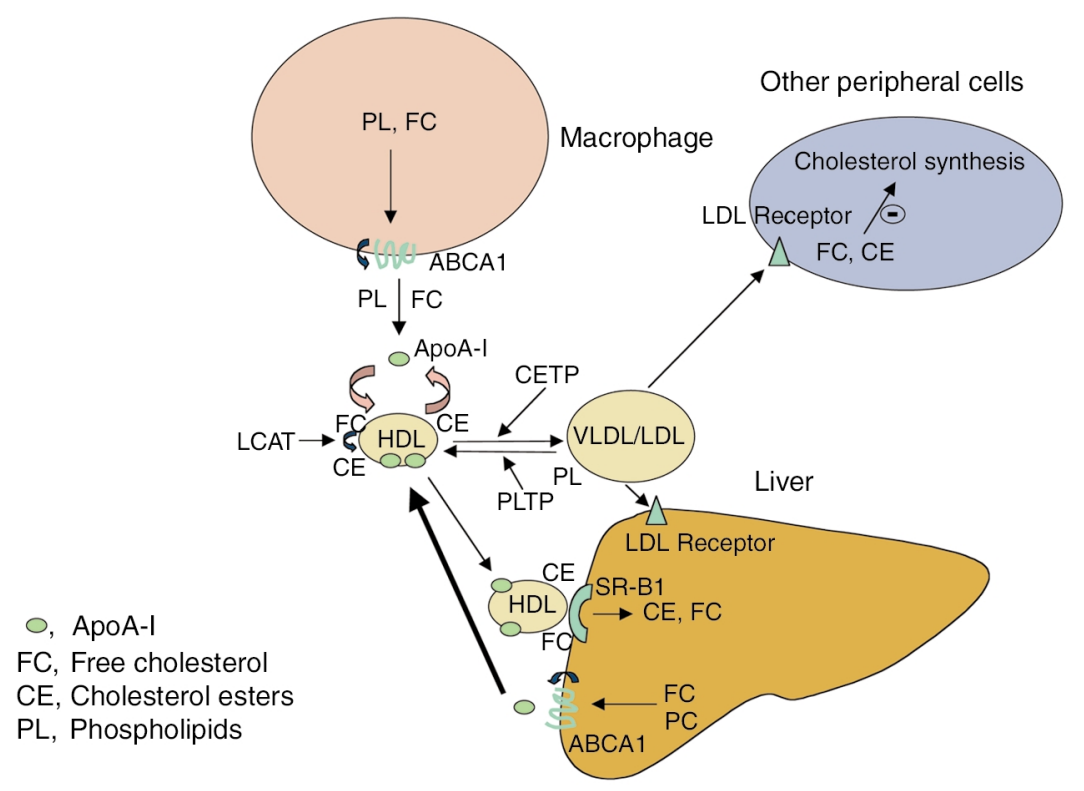

\section{Figure 1}

Contribution of ABCA1 to macrophage cholesterol efflux, HDL levels, and centripetal cholesterol flux. $A B C A 1$ probably has a major role in the efflux of phospholipids and cholesterol from different cells (macrophages, hepatocytes) to apoA-I. This is followed by further growth of the HDL particle, involving the addition of lipids and apoproteins, cholesterol esterification by lecithin-cholesterol acyltransfer (LCAT), and influx of phospholipids under the control of phospholipid transfer protein (PLTP), and eventuates in the formation of mature HDL. Macrophage ABCA1 makes only a minor contribution to the maintenance of circulating $\mathrm{HDL}$, while hepatic expression of $A B C A 1$ probably has a major role in this regard (thick arrow). Studies in ABCA1 KO mice indicate that although $A B C A 1$ has a key role in maintaining HDL levels, overall centripetal flow of cholesterol from periphery to liver appears not to be affected by the absence of ABCA1. Nonetheless, the phenotype of Tangier disease indicates that $A B C A 1$ has an essential role in removing cholesterol deposited in macrophages, but probably not other cells. The amount of cholesterol lost from macrophages is too small to be measured in the overall centripetal flow of cholesterol. Moreover, macrophagederived cholesterol could be redistributed to VLDL/LDL and taken up in other peripheral tissues, without substantially changing VLDL/LDL cholesterol levels or centripetal cholesterol flux. 
of any direct interaction with macrophage ABCA1. However, HDL can mediate macrophage cholesterol efflux by mechanisms unrelated to ABCA1 (9). Thus, hepatic and macrophage ABCA1 might act by distinct mechanisms to reduce the mass of cholesterol associated with macrophage foam cells. This might further lead to a decrease in the number of macrophages entering lesions, and hence lesion initiation or progression.

\section{No defect in reverse cholesterol transport detected in ABCA1 KO mice}

In a recent issue of the JCI, Groen et al. used ABCA1 $\mathrm{KO}$ mice to evaluate the role of ABCA1 in hepatic and biliary lipid metabolism and fecal sterol excretion (10). They found that biliary cholesterol, phospholipid, and bile salt secretion rates were similar in ABCA1 $\mathrm{KO}$ and control mice, whether these animals were fed regular chow or highfat, high-cholesterol diets. Moreover, fecal excretion of cholesterol and bile salts, and the proportion of newly synthesized cholesterol incorporated into biliary lipids, were unaltered in ABCA1 $\mathrm{KO}$ mice. These results are surprising because one might have expected that the very low HDL levels in ABCA1 KO mice would result in decreased delivery of cholesterol from plasma into liver and bile. The uptake of HDL free and esterified cholesterol via scavenger receptor B-I (SR-BI) has been shown to act as an important source of biliary cholesterol $(11,12)$. Of course, the decrease in hepatic cholesterol delivery seen in the HDL of ABCA1 KO mice might be compensated by other sources of cholesterol, either by an increase in hepatic cholesterol biosynthesis or by increased uptake of cholesterol from a non-HDL lipoprotein fraction. The authors attempted to measure these processes, first by monitoring levels of $\mathrm{mRNAs}$ for cholesterol biosynthetic enzymes, and second by following the clearance of artificial cholesterol/phospholipid vesicles in wild-type and ABCA1 KO mice. Neither test brought to light any significant compensatory mechanism in $\mathrm{KO}$ animals. Unfortunately, these experiments are not entirely satisfactory, since mRNA levels offer an imperfect gauge of cholesterol biosynthetic rates (13), and infusion of phospholipid vesicles may not adequately track the clearance of non-HDL cholesterol in ABCA1 KO mice. Nevertheless, the findings support the authors' conclusion that HDL levels do not control net cholesterol transport from the periphery via the liver into the bile (10).

The apparently modest contributions of macrophage cholesterol to HDL levels and of HDL to biliary cholesterol are at first glance surprising and provocative. However, these results are consistent with an important body of work from the laboratories of Spady and Dietschy (14-16). These studies have shown that over- or underexpression of individual molecules involved in the reverse cholesterol transport pathway does not perturb the centripetal flow of cholesterol from the periphery to the liver. Such studies have assessed overall reverse cholesterol transport by measuring peripheral cholesterol synthesis, which in the steady state approximates the centripetal flux of cholesterol. Unfortunately, they cannot provide information on efflux of cholesterol from specific cell types such as macrophages or smooth muscle cells in atheroma. These experiments have shown that in cholesteryl ester transfer protein transgenic, apoA-I KO, and SR-BI transgenic mice, all with reduced HDL levels, centripetal cholesterol flux is not appreciably changed (14-16). Similarly, overexpression of the plasma cholesterol-esterifying enzyme (LCAT), or of the rate-limiting enzyme of bile salt biosynthesis (Cyp7a), does not lead to a change in these measurements (14). Infusion of phospholipid/apoA-I discs, which are devoid of cholesterol and thus remove cholesterol from cells by passive or other mechanisms, likewise does not affect fecal sterol excretion, although it does lead to a temporary increase in reverse cholesterol transport to the liver (14).

These results indicate that overexpression of individual molecules in the chain of reverse cholesterol transport does not increase the efficiency of the overall process, suggesting that there is not a single, rate-limiting step. However, the simultaneous overexpression of multiple molecules involved in reverse cholesterol transport can increase fecal sterol excretion. Thus, adenoviral overexpression of human LCAT or human SR-BI individually in human apoA-I transgenic mice has only modest effects, whereas the com- bined overexpression of LCAT and SR-BI markedly increases fecal sterol excretion (17). There could also be important species differences. Infusion of phospholipid/pro-apoA-I discoidal HDL into four humans with heterozygous familial hypercholesterolemia $(\mathrm{FH})$ did increase biliary sterol excretion (18). However, careful human studies have shown that there is no relationship between plasma HDL levels and mathematically modeled tissue cholesterol pool sizes, suggesting that in humans HDL levels are not a general marker of the efficiency of centripetal cholesterol flux (19).

\section{Is it time to pull the plug on reverse cholesterol transport?}

Do these findings imply that we should throw out the idea that the protective role of HDL in atherogenesis is related to its ability to stimulate reverse cholesterol transport, or that increasing $\mathrm{ABCA} 1$ expression might be antiatherogenic? Not at all: While HDL levels may not determine the efficiency of centripetal cholesterol transport, the concentration of HDL or its apoproteins is certainly rate-limiting for macrophage cholesterol efflux. This is dramatically demonstrated by Tangier disease, where the cellular phenotype is a lack of phospholipid and cholesterol efflux to apoA-I (20), and macrophages but not other cells accumulate cholesteryl esters. Thus, the macrophage, a professional cholesterol scavenger, is particularly reliant on ABCA1 and apoA-I to rid itself of excess cholesterol, either by direct or by indirect mechanisms (Figure 1). The efflux of cholesterol from macrophage foam cells has not been measured in studies of centripetal cholesterol flux, or biliary lipid output. This contributes such a small fraction to the total cholesterol flux that it cannot be detected. Thus, an increase in apoA-I, HDL, or ABCA1 that leads to a decrease in macrophage foam cell cholesterol content is unlikely to change centripetal cholesterol flux in sufficient amounts to be detected. Increased cholesterol efflux from the macrophage could be followed by a small change in cholesterol transport to the liver, or even a slight increase in uptake in other peripheral tissues (Figure 1). Often the term "reverse cholesterol transport" is used to indicate the initial step in this process, i.e., the 
efflux of cholesterol from cells in atheroma (21). Perhaps we should stop using the term "reverse cholesterol transport" and replace it with more precise descriptors such as "macrophage cholesterol efflux" and "centripetal cholesterol transport."

The relationship between these processes requires further clarification. Plasma HDL levels may relate to macrophage cholesterol efflux, either by determining the efficiency of cholesterol removal from foam cells, or by acting as a marker of the level of ABCA1-mediated cholesterol efflux from foam cells, as in low HDL states associated with heterozygous ABCA1 mutations (22). It is now evident that plasma HDL level does not bear a simple relationship to centripetal cholesterol transport, but it cannot be excluded that some individuals with high HDL levels have increased centripetal cholesterol transport that includes an increase in macrophage cholesterol efflux.

\section{HDL as a therapeutic target}

The results of these studies suggest that it might be timely for lipoprotein researchers to place greater emphasis on the interaction of HDL and its apoproteins with cells in atheroma and to devote less effort pursuing the idea that centripetal cholesterol transport needs to be increased by therapeutic interventions involving HDL. Measurements of biliary lipid outputs or fecal sterol excretion may not provide any insight into the effectiveness of drugs that act on HDL to favorably affect foam cell cholesterol content and atherosclerosis. Successful interventions at the level of the foam cell could be mediated by increased ABCA1 expression (perhaps using the
LXR or RXR activators; refs. 2, 23), by increased apoA-I or HDL biosynthesis (which might be achieved via PPAR $\alpha$ activators), or by decreased HDL catabolism (using nicotinic acid or cholesteryl ester tramsfer protein [CETP] inhibitors). Finally, it is worth noting that small molecules that activate LXR or RXR act favorably to increase expression of several different molecules involved in macrophage cholesterol efflux and intestinal cholesterol excretion (24). Small-molecule activators of nuclear hormone receptors thus represent an attractive way to influence multiple steps of cholesterol homeostasis in a way that can reduce atherosclerosis $(3,23)$.

1. Schmitz, G., and Langmann, T. 2001. Structure, function and regulation of the $\mathrm{ABC} 1$ gene product. Curr. Opin. Lipidol. 12:129-140.

2. Repa, J.J., et al. 2000. Regulation of absorption and ABC1-mediated efflux of cholesterol by RXR heterodimers. Science. 289:1524-1529.

3. Costet, P., Luo, Y., Wang, N., and Tall, A.R. 2000 Sterol-dependent transactivation of the ABC1 promoter by the liver $\mathrm{X}$ receptor/retinoid $\mathrm{X}$ receptor. J. Biol. Chem. 275:28240-28245.

4. Scott, J. 1999. Heart disease. Good cholesterol news. Nature. 400:816-819.

5. McNeish, J., et al. 2000. High density lipoprotein deficiency and foam cell accumulation in mice with targeted disruption of ATP-binding cassette transporter-1. Proc. Natl. Acad. Sci. USA. 97:4245-4250.

6. Haghpassand, M., Bourassa, P.-A.K., Francone, O.L., and Aiello, R.J. 2001. Monocyte/ macrophage expression of ABCA1 has minimal contribution to plasma HDL levels. J. Clin. Invest. 108: $1315-1320$.

7. Vaisman, B.L., et al. 2001. ABCA1 overexpression leads to hyperalphalipoproteinemia and increased biliary cholesterol excretion in trans genic mice. J. Clin. Invest. 108:303-309.

8. Wang, N., Silver, D.L., Costet, P., and Tall, A.R. 2000. Specific binding of ApoA-I, enhanced cholesterol efflux, and altered plasma membrane morphology in cells expressing ABC1. J. Biol. Chem. 275:33053-33058.

9. Rothblat, G.H., et al. 1999. Cell cholesterol efflux: integration of old and new observations provides new insights. J. Lipid Res. 40:781-796.

10. Groen, A.K., et al. 2001. Hepatobiliary cholesterol transport is not impaired in Abca1-null mice lacking HDL. J. Clin. Invest. 108:843-850.
11.Ji, Y., et al. 1999. Hepatic scavenger receptor BI promotes rapid clearance of high density lipoprotein free cholesterol and its transport into bile. J. Biol. Chem. 274:33398-33402.

12. Mardones, P., et al. 2001. Hepatic cholesterol and bile acid metabolism and intestinal cholesterol absorption in scavenger receptor class B type Ideficient mice. J. Lipid Res. 42:170-180.

13. Shimomura, I., Bashmakov, Y., and Horton, J.D. 1999. Increased levels of nuclear SREBP-1c associated with fatty livers in two mouse models of diabetes mellitus. J. Biol. Chem. 274:30028-30032.

14. Alam, K., Meidell, R.S., and Spady, D.K. 2001. Effect of up-regulating individual steps in the reverse cholesterol transport pathway on reverse cholesterol transport in normolipidemic mice. $J$. Biol. Chem. 276:15641-15649.

15. Osono, Y., Woollett, L.A., Marotti, K.R., Melchior, G.W., and Dietschy, J.M. 1996. Centripetal cholesterol flux from extrahepatic organs to the liver is independent of the concentration of high density lipoprotein-cholesterol in plasma. Proc. Natl. Acad. Sci. USA. 93:4114-4119.

16. Jolley, C.D., Woollett, L.A., Turley, S.D., and Dietschy, J.M. 1998. Centripetal cholesterol flux to the liver is dictated by events in the peripheral organs and not by the plasma high density lipoprotein or apolipoprotein A-I concentration. J. Lipid Res. 39:2143-2149.

17. Mucksavage, P., et al. 2001. Co-expression of LCAT and SR-bi markedly increases fecal sterol excretion as a marker of reverse cholesterol transport; In Second Annual Conference on Arteriosclerosis, Thrombosis and Vascular Biology Arterioscler. Thromb. Vasc. Biol. 21:652. Abstr.

18. Eriksson, M., Carlson, L.A., Miettinen, T.A., and Angelin, B. 1999. Stimulation of fecal steroid excretion after infusion of recombinant proapolipoprotein A-I. Potential reverse cholesterol transport in humans. Circulation. 100:594-598.

19. Blum, C.B., et al. 1985. Relationship of the parameters of body cholesterol metabolism with plasma levels of HDL cholesterol and the major HDL apoproteins. J. Lipid Res. 26:1079-1088.

20. Oram, J.F., and Yokoyama, S. 1996. Apolipoprotein-mediated removal of cellular cholesterol and phospholipids. J. Lipid Res. 37:2473-2491.

21. Tall, A.R., and Wang, N. 2000. Tangier disease as a test of the reverse cholesterol transport hypothesis. J. Clin. Invest. 106:1205-1207.

22. Clee, S.M., et al. 2000. Age and residual cholesterol efflux affect HDL cholesterol levels and coronary artery disease in ABCA1 heterozygotes. J. Clin. Invest. 106:1263-1270.

23. Claudel, T., et al. 2001. Reduction of atherosclerosis in apolipoprotein $\mathrm{E}$ knockout mice by activation of the retinoid X receptor. Proc. Natl. Acad. Sci. USA. 98:2610-2615.

24. Tall, A.R., Costet, P., and Luo, Y. 2000. 'Orphans' meet cholesterol. Nat. Med. 6:1104-1105. 